\title{
Visual and proprioceptive representations in spatial memory
}

\author{
NAOHIDE YAMAMOTO and AMY L. SHELTON \\ Johns Hopkins University, Baltimore, Maryland
}

\begin{abstract}
It has been shown that spatial information can be acquired from both visual and nonvisual modalities. The present study explored how spatial information from vision and proprioception was represented in memory, investigating orientation dependence of spatial memories acquired through visual and proprioceptive spatial learning. Experiment 1 examined whether visual learning alone and proprioceptive learning alone yielded orientation-dependent spatial memory. Results showed that spatial memories from both types of learning were orientation dependent. Experiment 2 explored how different orientations of the same environment were represented when they were learned visually and proprioceptively. Results showed that both visually and proprioceptively learned orientations were represented in spatial memory, suggesting that participants established two different reference systems based on each type of learning experience and interpreted the environment in terms of these two reference systems. The results provide some initial clues to how different modalities make unique contributions to spatial representations.
\end{abstract}

Many human activities require information about the locations of objects in an environment: retrieving a key from a desk drawer, walking to a closet to pick up a jacket, and navigating to a mailroom to find a piece of mail, among others. All these actions require understanding the locations of target objects. This information tends to be regarded as visually acquired, but spatial information from multiple modalities can also be used to guide behavior. For example, when reaching for a ringing phone that is placed on the far side of a room, one may turn to see the phone. Alternatively, the sound of the ringing may provide sufficient information for walking to the right location. As one approaches the phone, the moving body provides proprioceptive feedback about the distance and direction to it. Touching the handset then provides tactile cues that can be used to establish the exact place to hold.

Many previous studies have demonstrated that spatial information can be acquired from nonvisual modalities, such as audition (Dufour, Després, \& Pebayle, 2002; Klatzky, Lippa, Loomis, \& Golledge, 2002; Loomis, Klatzky, Philbeck, \& Golledge, 1998), taction (Klatzky, 1999; Klatzky \& Lederman, 2003; Shelton \& McNamara, 2001b), and proprioception (Chance, Gaunet,

Portions of this research were presented at the 11th annual workshop on Object Perception, Attention, \& Memory in Vancouver, Canada (November 2003). N.Y. was supported by a graduate study fellowship from the Nakajima Foundation. We thank Michael McCloskey, David Waller, and two anonymous reviewers for their invaluable comments on earlier versions of the manuscript. Correspondence should be addressed to N. Yamamoto, Department of Psychological and Brain Sciences, Johns Hopkins University, 3400 N. Charles Street, Baltimore, MD 21218 (e-mail: yamamoto@jhu.edu).
Beall, \& Loomis, 1998; Klatzky, Loomis, Beall, Chance, \& Golledge, 1998; Waller, Loomis, \& Haun, 2004). It has also been shown that these types of information are available both in small-scale spaces (Klatzky, 1999; Klatzky \& Lederman, 2003; Shelton \& McNamara, 2001b) and in large-scale spaces (Loomis et al., 1998; Waller et al., 2004). The present study focused on the effects of vision and proprioception on the learning of a room-sized spatial layout. By having participants learn a layout of objects both by vision and by proprioception, the multimodal nature of spatial memory was investigated. In this article, proprioception was broadly defined as the sense of body movement mediated by information generated inside the body. This information includes sensory signals about the motion of body parts provided by receptors in muscles, tendons, and joints (proprioception proper), sensation of linear and angular accelerations produced by the vestibular system, and efference copies of motor commands generated by the central nervous system.

Because visual information is generally available for spatial learning in everyday environments, many previous studies on proprioceptive spatial learning have investigated the contribution of proprioception in addition to that of vision. For example, Chance et al. (1998) and Waller et al. (2004) have explored how proprioceptive information about a large-scale environment facilitated the retrieval of memories for the locations of landmarks. Both studies commonly had two types of experimental conditions. In one condition (the "walk" condition), participants acquired proprioceptive information about the environments by walking around them. While walking, they experienced the environments visually as well. In the other conditions, participants did not receive propri- 
oceptive information by sitting in a chair (the "sit" and "smooth" conditions in Waller et al., 2004) or standing still (the "visual turn" condition in Chance et al. 1998), but they were provided with the same visual information as in the walk condition. Participants pointed to the landmarks more accurately in the walk condition than in the other conditions, showing the benefits of having access to proprioceptive information about the environments. However, these studies were not designed to discern a unique effect of proprioceptive information, because it was always accompanied by visual information. In the present study, we tried to separate the contribution of proprioception from that of vision by asking participants to learn a layout separately through vision and proprioception.

The present study was also inspired by the theory of spatial memory proposed by McNamara and colleagues (Mou \& McNamara, 2002; Shelton \& McNamara, 2001a; see also Werner \& Schmidt, 1999). According to this theory, learning the locations of objects in a new environment involves first setting up a spatial reference system. In a similar manner to determining the "top" of a figure in form perception (Rock, 1973), the first step is to establish a conceptual "north" that provides an intrinsic reference system by which the locations of objects are defined. This process gives rise to preferred orientations of the environment in which spatial learning and memory have benefits (e.g., an advantage in retrieval of spatial information). The intrinsic reference system can be defined by various egocentric and environmental cues, such as egocentric views (Shelton \& McNamara, 1997, 2001a), local and global structures of a surrounding environment (McNamara, Rump, \& Werner, 2003; Shelton \& McNamara, 2001a), and instruction (Mou \& McNamara, 2002). As such, there are many potential intrinsic reference axes for a given environment, but some are more likely to be selected because of their salience. Unless exceptionally salient environmental cues are available, egocentric experience provides the most salient orientation, and the environment is interpreted in terms of the spatial reference system defined by the corresponding intrinsic axis.

In the context of the present study, this theory poses a question as to how an intrinsic reference axis is selected when participants learn a layout separately through vision and proprioception. Because both visual and proprioceptive learning provide participants with egocentric experiences of the layout, two intrinsic axes based on each type of learning will be more salient than others. It is not clear, however, which of these two axes the participants will select to interpret the layout. If one is more salient than the other, the more salient axis will be selected. It is also possible that the modalities do not differ in salience. In this case, the axis corresponding to the first experienced orientation (either visually or proprioceptively) may be selected, as has been shown for the case in which multiple orientations of a layout were experienced visually (Shelton \& McNamara, 2001a). ${ }^{1}$ Alternatively, the two axes corresponding to different modal- ities may be more independent from each other than axes corresponding to the same modality, and therefore both the axis based on visual learning and the axis based on proprioceptive learning may be selected to set up the spatial reference systems.

In order to explore which type of learning provides an intrinsic reference axis, we examined the orientation dependence of spatial memory by employing a research paradigm established by Shelton and McNamara (1997, 2001a, 2001b, 2004; for a similar methodology, see also Presson \& Hazelrigg, 1984). Spatial memory is said to be orientation dependent when particular perspectives (preferred orientations) are more accessible than other perspectives (nonpreferred orientations). It is assumed that preferred orientations are explicitly represented in memory by the intrinsic reference axes, whereas nonpreferred orientations must be inferred by mentally transforming spatial information. This mental transformation may be performed by rotating and/or translating either an egocentric viewpoint relative to a stationary environment or the entire environment relative to a stationary egocentric viewpoint (Zacks, Mires, Tversky, \& Hazeltine, 2000). Although different types of mental spatial transformations are possible, the essential point is that any mental transformation should produce measurable costs in terms of latency and/or error during memory access. Retrieval of spatial relations aligned with preferred orientations is therefore faster and/or more accurate than retrieval of spatial relations aligned with nonpreferred orientations, thereby indicating which intrinsic axis (or axes) is selected to represent the environment.

If different orientations of the same environment are learned visually, a specific learned orientation tends to be preferred in subsequent spatial memory (Shelton \& McNamara, 2001a). Given that learning multiple orientations of the same spatial layout tends to produce a single preferred orientation in memory, we can explore what happens when those learned orientations are encoded by different modalities. For example, previous studies employed this paradigm when the different orientations were learned either through vision and taction (Shelton \& McNamara, 2001b) or through vision and verbal description (Shelton \& McNamara, 2004), showing the preference for both the visually learned orientations and the nonvisually learned orientations. In the present study, we asked whether a visually learned orientation, a proprioceptively learned orientation, or both orientations were preferred during memory access. It was also possible that the first learned orientation was preferred, regardless of the modality through which it was learned.

To effectively apply Shelton and McNamara's paradigm for the present study, we first needed to determine whether both visual learning alone and proprioceptive learning alone yield orientation-dependent spatial memory. The theoretical framework described above predicted that each type of learning would produce orientationdependent spatial memory, and in fact, it has been well documented that visually acquired memories for a room- 
sized spatial layout are orientation dependent (RoskosEwoldsen, McNamara, Shelton, \& Carr, 1998; Shelton \& McNamara, 1997, 2001a; Sholl \& Nolin, 1997; Waller, Montello, Richardson, \& Hegarty, 2002; but see also Presson, DeLange, \& Hazelrigg, 1989; Presson \& Hazelrigg, 1984; Sholl \& Nolin, 1997). However, few studies have investigated whether spatial memories learned by proprioception alone are orientation dependent. Presson, DeLange, and Hazelrigg (1987) conducted an experiment related to this specific question, demonstrating that spatial memories learned by nonsighted locomotion were orientation dependent when participants experienced only a single orientation during learning (the "walk-no turn" condition). In their experiment, blindfolded participants learned various paths in a room by walking on them. Each path consisted of three segments connecting four locations. They maintained a constant orientation during walking by taking side, diagonal, and back steps when needed. After having learned a path, they were taken to one of the four locations on the path and asked to make directional judgments - for example, "You are at Location 1, and Location 2 is directly in front of you. Point to where Location 3 is" (Presson et al., 1987, p. 227). Participants made more accurate directional judgments when they were in the learned orientation than when they were in a novel orientation that was opposite to the learned orientation, showing that their spatial memories were orientation dependent.

Although Presson et al.'s (1987) results showed the orientation dependence of spatial memory acquired by proprioception alone, the restricted conditions used in their experiment limited the investigation. Presson et al. (1987) compared participants' preference for orientations only between the learned orientation and the novel orientation that was the direct opposite of the learned orientation. As McNamara (2003) pointed out, a certain amount of benefit for the retrieval of spatial memory is often observed for the opposite orientation. Thus, comparing the preference for the learned orientation only with that for the opposite orientation is not an ideal way to investigate orientation dependence of spatial memory. It is impressive that Presson et al. (1987) found orientationdependent spatial memory in spite of this limitation, but this result called for further investigation in which the preference for the learned orientation is compared with that for novel orientations with systematically varying angular distance between these two types of orientations.

In the present study, therefore, we first conducted an experiment to investigate whether spatial memory for a room-sized layout acquired from proprioception alone would be orientation dependent, when angular distance between learned and novel orientations was systematically varied. Second, we explored how spatial information from visual and proprioceptive learning was represented in memory by having participants learn different orientations of the same layout, one visually and the other proprioceptively.

\section{EXPERIMENT 1}

The objective of Experiment 1 was to determine whether spatial memories for a room-sized layout of objects acquired through proprioception alone were orientation dependent, thereby establishing a foundation for applying Shelton and McNamara's paradigm to the present study. Subsequent spatial memories were tested after participants learned each of two different layouts, one by vision and the other by proprioception.

\section{Method}

Participants. Twenty-four participants from the Johns Hopkins community (12 males and 12 females, ranging in age from 18 to 22) volunteered in return for extra credit in psychology courses.

Materials and Design. Two layouts of six objects each were constructed. The objects were common, visually distinct, and similar in

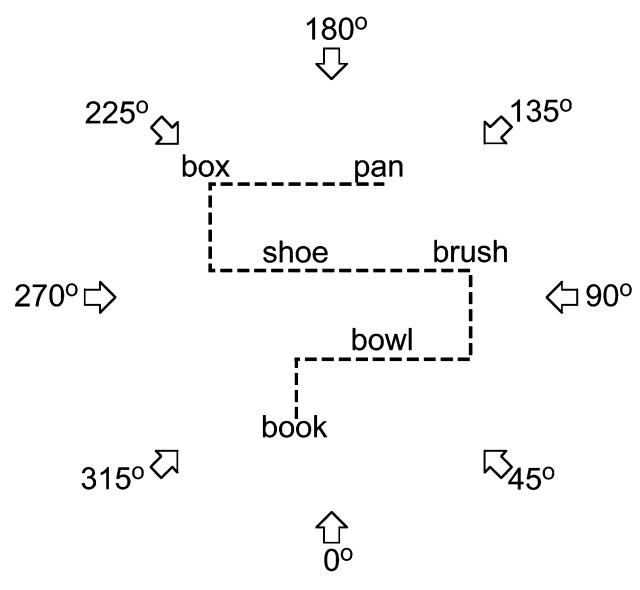

Layout A

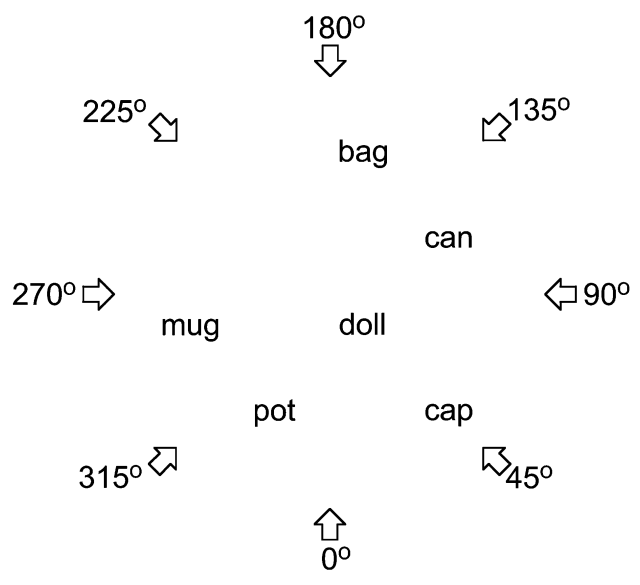

Layout B

Figure 1. An example of each layout used in the experiments. Real objects were used, not names only. The dashed line on Layout A shows an example of a walking path taken in proprioceptive learning. Counterbalancing was used to ensure that each layout was experienced both visually and proprioceptively. 
size (approximately $15 \mathrm{~cm}$ in length and width, and $10 \mathrm{~cm}$ in height). Each layout had three variations; the configuration remained unchanged, but the arrangement of six objects was different among these variations. An example of each layout is shown in Figure 1. The objects were placed on a plain white sheet, which was laid on the floor of an approximately $3 \times 3.7-\mathrm{m}$ room. A white curtain created a uniform texture around the walls of the room in order to minimize the participants' use of external cues in spatial learning.

All participants learned both layouts: One was learned visually and the other proprioceptively. Half of the participants learned Layout A through vision and Layout B through proprioception. The other half learned Layout A through proprioception and Layout B through vision. The different versions of each layout were counterbalanced. The order of visual and proprioceptive learning was also counterbalanced.

After each learning phase, the participants performed judgments of relative direction (JRDs) to assess their spatial memories for the layouts. Three objects in a layout formed each trial: The participants were asked to imagine themselves standing at one object and facing another object. They were then asked to point to the third object; for example, "Imagine you are at the box and facing the pan. Point to the brush." The first two objects constituted an imagined heading. The third was a target.

The primary independent variable was the imagined heading. Each object-pair represented one of eight imagined headings differing by $45^{\circ}$. Each layout yielded 16 object-pair headings, two instances of each of the eight imagined headings. These imagined headings were labeled counterclockwise from $0-315^{\circ}$ in $45^{\circ}$ steps, with $0^{\circ}$ corresponding to the orientation learned by the participants. Target objects were chosen so that their directions were varied systematically. The egocentric space was divided into four homogeneous regions (i.e., front, back, left, and right), and each imagined heading had approximately equal instances of each target direction that lay in one of these four regions.

Procedure. Four groups were formed from the factorial combination of layout (Layout A for visual learning and Layout B for proprioceptive learning, or the opposite combination) and order of learning (visual learning first or proprioceptive learning first). The participants were randomly assigned to each group with the constraint that each group had an equal number of males and females. The participants were run individually.

Learning phase. In the beginning of visual learning, the participants were asked to sit in a caster chair and wear a blindfold before they went into a room in which objects were placed. The blindfold completely restricted the participants' vision. An experimenter pushed the chair and brought the participants to the position from which they viewed the layout. (This position is labeled as $0^{\circ}$ in Figure 1.) While the participants were taken to the viewing position, the chair was gently spun by the experimenter to disorient them. This disorienting procedure was included in order to prevent the participants from using environmental cues for spatial learning. (The effectiveness of this procedure was confirmed by a separate experiment. ${ }^{2}$ ) At the viewing position, the participants were asked to get up from the chair and remove the blindfold. The participants learned the layout by viewing it for $30 \mathrm{sec}$, after which they were asked to close their eyes and point to and name each object. This study-test sequence was repeated until the participants were able to fluently point to and name each object correctly twice in a row. (Fluency was determined by the experimenter's discretion based on no hesitation in pointing.) After the participants successfully finished this study-test sequence, they were offered additional viewing time, although only a few participants took it. The participants were then asked to put the blindfold back on and sit in the chair again. The experimenter pushed the chair and took the participants out to the next room, turning the chair to disorient them.

In proprioceptive learning, the participants were wheeled into the room in the same manner as in visual learning. On arriving at the start point, the participants were asked to get up from the chair. (The start point is labeled as $0^{\circ}$ in Figure 1.) Unlike in visual learning, the participants kept wearing the blindfold throughout the learning phase. They then walked to the end point by way of each object (see Figure 1). The participants held a bar horizontally with both hands, and the experimenter pulled the bar to indicate the distance and direction of their walking. In addition, the participants were guided verbally (e.g., walk forward, leftward; stop now). The participants were asked to maintain the orientation that they had at the start point: They took side steps for rightward and leftward walking, and back steps for backward walking. The participants' walking included movement parallel to the $90-270^{\circ}$ axis in this manner, but the proprioceptively learned orientation was defined by their facing direction $\left(0^{\circ}\right)$. Upon reaching each object, the participants viewed the object briefly by opening a narrow gap in the blindfold, seeing its identity and appearance. ${ }^{3}$ Because the blindfold was designed to allow the participants to see only a small area around their feet (approximately $30 \mathrm{~cm}$ in width and depth), they were unable to have direct visual access to the entire layout. At the last object, the participants were guided back to the start point following the same path in the backward direction. When the participants returned to the start point, they were asked to point to and name each object. This study-test sequence was repeated until the participants reached the same criterion used in visual learning. After the participants successfully finished this study-test sequence, they were offered an opportunity for an additional walking, but only a few participants walked one more time. The participants were then asked to sit in the chair and were disoriented while taken out of the room.

Test phase. After each learning phase, the participants were taken to another room to perform JRDs. Trials were presented on a computer screen. After receiving instructions about how to use a computer program, the participants performed three practice trials involving buildings on campus. In each trial, a sentence giving an imagined heading and target was displayed with a circle and a movable line. The participants positioned the line by using a mouse so that it pointed to the target if they were at the imagined position. There were 48 trials presented in one block. The order of imagined headings and target directions was randomized. Absolute angular error in pointing (i.e., absolute angular distance between pointed direction and target direction) and response latency was recorded in each trial. The participants were asked to answer as accurately as possible, but the task was not speeded.

\section{Results and Discussion}

In this and subsequent experiments, both angular error in pointing and response latency showed the same general pattern, but the effects shown by response latency were smaller and more variable than those of angular error. Hence only angular error is discussed in detail. There was no evidence of speed-accuracy tradeoff. (The correlation coefficient between absolute angular error and response latency was .32.) Mean response latencies were $16.63 \mathrm{sec}$ and $16.86 \mathrm{sec}$ in visual and proprioceptive learning, respectively.

One data point was excluded from the analyses because of its extremely short response latency $(74 \mathrm{msec})$. Means were then calculated for each participant and for each condition. Absolute angular error was analyzed by a split-plot factorial analysis of variance (ANOVA) with layout (Layout A for visual learning and Layout B for proprioceptive learning, or the opposite combination), order of learning (visual learning first or proprioceptive learning first), and gender (male or female) as betweenparticipants factors and imagined heading (from $0-315^{\circ}$ with $45^{\circ}$ intervals), target direction (front, back, left, and 
right), and modality of learning (vision and proprioception) as within-participants factors. Due to the large number of possible main effects and interactions, an $\alpha$ level of .01 was used.

Figure 2A shows mean absolute angular error in pointing collapsed across participants as a function of imagined heading and modality of learning, revealing two major results. First, participants were most accurate in pointing when imagined heading corresponded to the learned orientation $\left(0^{\circ}\right)$. This was supported statistically by the significant main effect of imagined heading $[F(7,112)=6.23]$, and a post hoc contrast comparing performance for the imagined heading of $0^{\circ}$ with that for other imagined headings $[F(1,23)=23.18]$. Second, participants' performances on JRDs after each type of learning were very similar to each other, as indicated by the fact that the data from each type of learning showed a similar variation pattern in Figure 2A. Neither the main effect of modality of learning nor interactions including this factor were significant. These results are more clearly captured by Figure 2B, showing mean absolute angular error in pointing as a function of modality of learning and type of orientation (learned orientation or novel orientations).

The ANOVA also revealed the significant main effect of target direction $[F(3,48)=10.25]$. As in previous studies (Shelton \& McNamara, 1997, 2001a, 2004; see also Franklin, Henkel, \& Zangas, 1995), pointing to the front was more accurate than pointing to the side, and pointing to the side was more accurate than pointing to the back. Mean absolute angular errors in pointing $(M)$ and corresponding standard errors of the mean (SEM) were: for pointing to the front, $M=31.11^{\circ}, S E M=1.63^{\circ}$; for pointing to the side, $M=34.58^{\circ}, S E M=1.07^{\circ}$; for pointing to the back, $M=42.46^{\circ}, S E M=1.72^{\circ}$. A post hoc contrast comparing accuracy in pointing to the back with that to all the other directions was significant $[F(1,23)=$ 16.97]. This main effect did not alter the conclusions about imagined heading.

These results clearly indicate that spatial memories from proprioceptive learning as well as those from visual learning were orientation dependent. After both types of learning, JRDs were more accurate for the learned orientations than for the novel orientations.

A

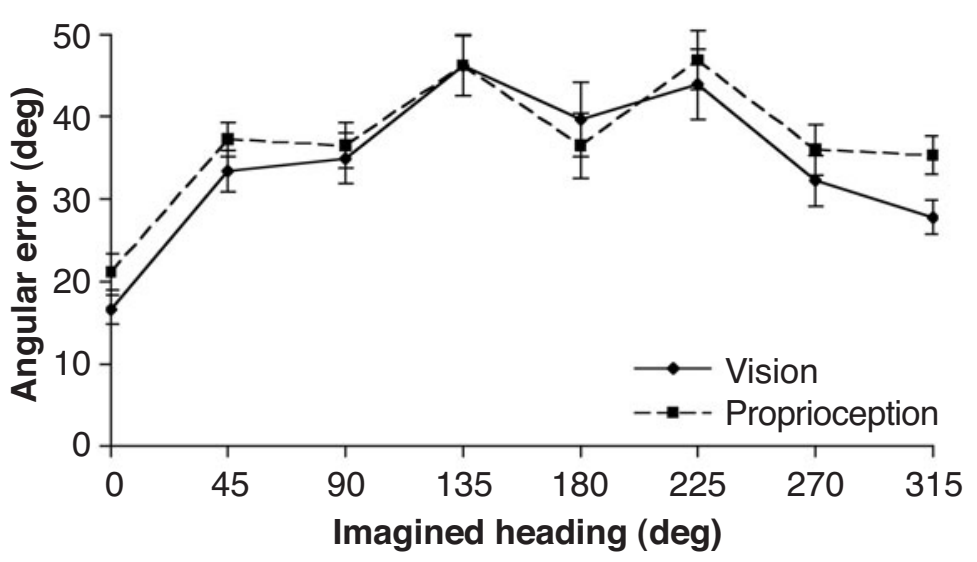

B

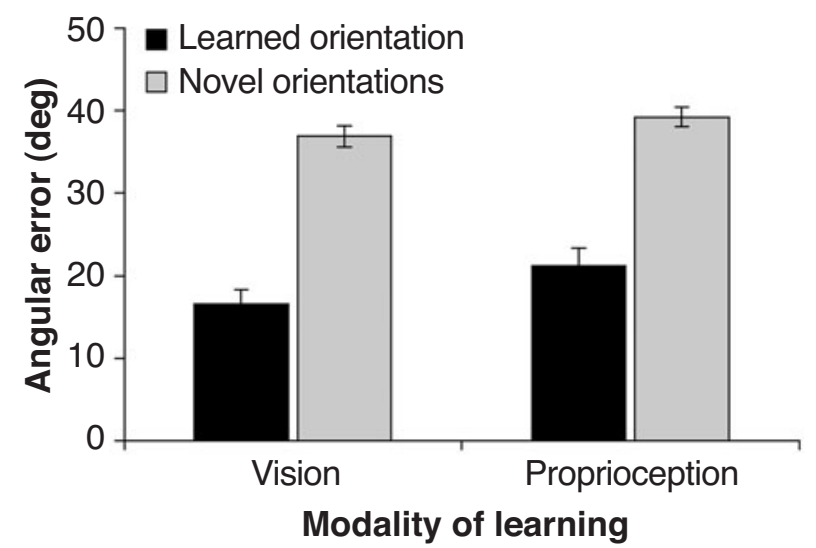

Figure 2. Mean absolute angular error in judgments of relative direction in Experiment 1 as a function of (A) imagined heading and modality of learning and $(B)$ type of orientation and modality of learning. Error bars represent \pm 1 SEM. 
Given the purpose of Experiment 1, the result that visual and proprioceptive learning yielded comparable spatial memories is also notable: Accuracy in JRDs varied similarly with imagined heading after each type of learning. Spatial memories from each type of learning were almost indistinguishable in this manner, suggesting that Shelton and McNamara's paradigm would work quite efficiently for the present study.

\section{EXPERIMENT 1A}

In the proprioceptive learning employed in Experiment 1 , participants viewed each object briefly by opening a narrow gap in the blindfold. Although their visual access to the entire layout was blocked, it was possible that these small portions of visual information confounded the effect of proprioception on spatial learning with that of vision. To examine this possibility, participants in Experiment 1A proprioceptively learned the layouts as in Experiment 1, except they had no visual information during walking.

\section{Method}

Participants. Eleven participants from the Johns Hopkins community (5 males and 6 females, ranging in age from 18 to 21) volunteered in return for extra credit in psychology courses.

Materials and Design. The objects were placed in the room that was used in Experiment 1. Each participant learned a single layout proprioceptively. Five participants learned Layout A, and 6 participants learned Layout B (see Figure 1). The participants' memories for the layout were assessed with JRDs.

Procedure. Two groups were formed on the basis of the layout that each participant learned. The participants were randomly assigned to each group with the constraint that each group had a nearly equal number of males and females ( 2 males and 3 females in one group; 3 males and 3 females in the other group). The participants were run individually.

Learning phase. The procedure for proprioceptive learning was the same as in Experiment 1 except for the following differences. Before the participants put on the blindfold, an experimenter showed them individual objects that constituted a layout. After this initial viewing, the participants were not allowed to see the objects.
Every time the participants reached an object in the course of walking, the experimenter asked them to stop and told them which object was at their feet. The experimenter used each object's name for this purpose.

Test phase. The participants performed JRDs in the same manner as in Experiment 1.

\section{Results and Discussion}

Absolute angular error was analyzed by a split-plot factorial ANOVA with layout (Layout A or Layout B) and gender (male or female) as between-participants factors and imagined heading (from $0-315^{\circ}$ with $45^{\circ}$ intervals) and target direction (front, back, left, and right) as withinparticipant factors. An $\alpha$ level of .05 was used because of the relatively small number of possible main effects and interactions.

Figure 3 shows mean absolute angular error in pointing, collapsed across participants as a function of imagined heading. The data resembled those from proprioceptive learning in Experiment 1. Figure 3 shows that participants were most accurate in JRDs when the imagined heading corresponded to the learned orientation $\left(0^{\circ}\right)$. The ANOVA revealed the significant main effect of imagined heading $[F(7,49)=3.46]$.

For comparison between the original and modified versions of proprioceptive learning, the data from this experiment were compared with those from the proprioceptive learning in Experiment 1. A split-plot factorial ANOVA was conducted with experiment (Experiment 1 or Experiment $1 \mathrm{~A})$ as a between-participants factor and imagined heading (from $0-315^{\circ}$ with $45^{\circ}$ intervals) as a withinparticipants factor. This ANOVA showed that neither the main effect of experiment nor the interaction between experiment and imagined heading was significant $[F(1,33)=$ 0.49 and $F(7,231)=1.89$, respectively]. The main effect of imagined heading was significant $[F(7,231)=7.36]$.

Mean response latency was $19.59 \mathrm{sec}$. There was no evidence for speed-accuracy tradeoff. (The correlation coefficient between absolute angular error and response latency was .13.)

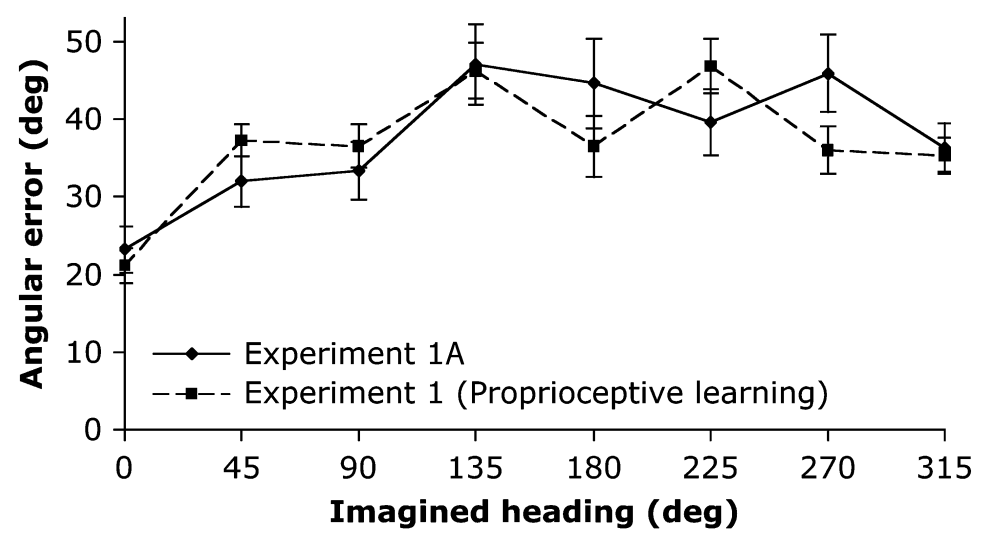

Figure 3. Mean absolute angular error in judgments of relative direction in Experiment $1 \mathrm{~A}$ as a function of imagined heading. For ease of comparison, data from proprioceptive learning in Experiment 1 are replicated here. Error bars represent $\pm 1 S E M$. 
In sum, the modified version of proprioceptive learning in which participants had no visual information during walking yielded essentially the same spatial memory as that from the proprioceptive learning used in Experiment 1 . Although participants recognized objects by receiving verbal information instead of visual information, it is unlikely that this verbal information led to different mental representations of the layouts. Thus, the results indicate that the effect of proprioception on learning a layout was not confounded with visual information coming from briefly viewing each object in the course of proprioceptive learning.

\section{EXPERIMENT 2}

On the basis of the findings from Experiments 1 and 1B, we investigated in Experiment 2 whether a visually learned orientation, a proprioceptively learned orientation, both orientations, or the first learned orientation would be preferred during memory access when a single layout was learned in two modalities.

\section{Method}

Participants. Forty-eight students at Johns Hopkins University (24 males and 24 females ranging in age from 17 to 25) participated in return for monetary compensation or extra credit in psychology courses.

Materials and Design. The objects were placed in the same room as in Experiment 1. Each participant learned two orientations of a single layout: One was learned visually, and the other was learned proprioceptively. Half of the participants learned Layout A, and the other half learned Layout B (see Figure 1). The viewing position for visual learning was randomly chosen from eight equally spaced positions indicated by arrows in Figure 1, having the constraint that half of the visually learned orientations were aligned with walls of the room, and the other half were misaligned with them. Three angular disparities between the visually learned orientation and the proprioceptively learned orientation were used: $0^{\circ}, 90^{\circ}$, or $135^{\circ}$. For $0^{\circ}$, the visually and proprioceptively learned orientations were identical. For $90^{\circ}$ and $135^{\circ}$, leftward and rightward disparities were counterbalanced. When the disparity was $90^{\circ}$, both the visually and proprioceptively learned orientations were either aligned with the walls or misaligned with them. When the disparity was $135^{\circ}$, one orientation was aligned with the walls, and the other was misaligned with them. The start point for proprioceptive learning was indicated by an arrow on the floor so that the participants were aware of the spatial relationship between the viewing position and the start point. Half of the participants did visual learning first, and the other half did proprioceptive learning first. Memory performance was examined with JRDs. Because the objective of this experiment was to explore which learned orientation(s) would be preferred during memory access, the primary independent variable here was type of orientation (visually learned orientation, proprioceptively learned orientation, and novel orientations).

Procedure. Twenty-four groups were formed by factorially combining order of learning (visual learning first or proprioceptive learning first), angular disparity between two learned orientations $\left(0^{\circ}, 90^{\circ}\right.$, or $\left.135^{\circ}\right)$, alignment (whether or not the visually learned orientation was aligned with walls), and direction of angular disparity (leftward or rightward). The participants were randomly assigned to each group with the constraint that each group had 1 male and 1 female. Each group also contained equal instances of each layout (Layout A or Layout B). The participants were run individually.

Learning phase. Each type of learning was done in the same way as in Experiment 1, except for the following difference: After the participants finished the first type of learning, they were asked to put on the blindfold (if they first learned the layout visually) and sit in the caster chair. They were then wheeled to the position from which they did the second type of learning.

Test phase. The participants performed JRDs (and scene recognition; see note 3 ) in the same manner as in Experiment 1.

\section{Results and Discussion}

Absolute angular error was analyzed by a split-plot factorial ANOVA with order of learning (visual learning first or proprioceptive learning first), angular disparity between two learned orientations $\left(0^{\circ}, 90^{\circ}\right.$, or $\left.135^{\circ}\right)$, alignment (whether or not the visually learned orientation was aligned with walls), and direction of angular disparity (leftward or rightward) as between-participants factors and type of orientation (visually learned orientation, proprioceptively learned orientation, and novel orientations) and target direction (front, back, left, and right) as within-participants factors. ${ }^{4}$ An $\alpha$ level of .01 was used because of the large number of possible main effects and interactions.

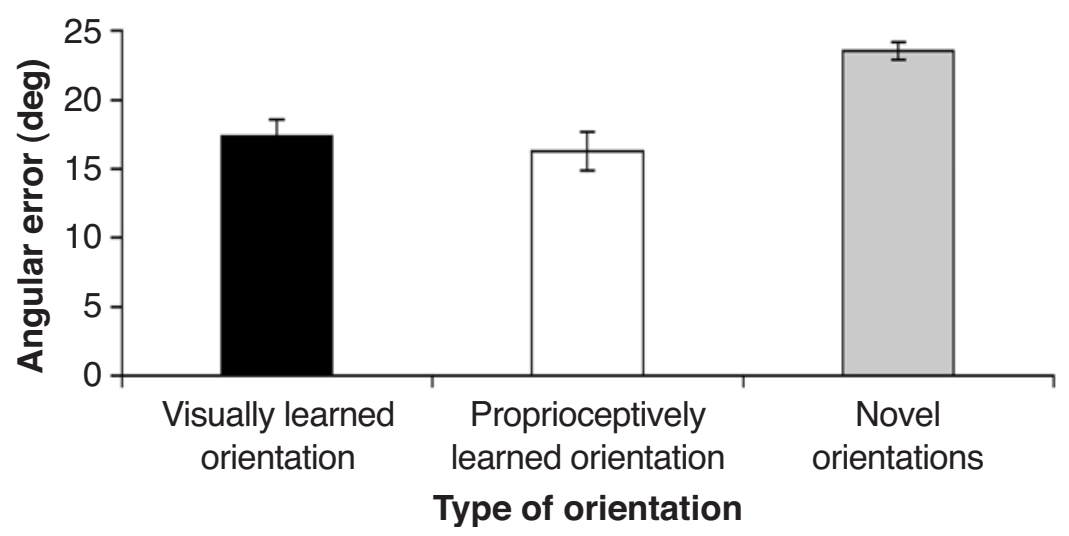

Figure 4. Mean absolute angular error in judgments of relative direction in Experiment 2 as a function of type of orientation. Error bars represent $\pm 1 S E M$. 
Mean absolute angular error in pointing, collapsed across participants, is plotted in Figure 4 as a function of type of orientation. JRDs were more accurate for both types of learned orientations than for novel orientations. This was supported statistically by the significant main effect of type of orientation $[F(2,48)=11.68]$, and a post hoc contrast comparing the two types of learned orientations with novel orientations $[F(1,47)=34.17]$. Figure 4 also suggests that the benefit for each type of learned orientation relative to novel orientations was equivalent. A post hoc contrast comparing the visually learned orientation and the proprioceptively learned orientation was not significant $[F(1,47)=0.35]$.

The ANOVA also revealed that the main effect of target direction was significant $[F(3,72)=8.18]$. As in Experiment 1 , pointing to the front was more accurate than pointing to the side, which was more accurate than pointing to the back. Mean absolute angular errors in pointing and corresponding standard errors of the mean were: for pointing to the front, $M=16.38^{\circ}, S E M=0.78^{\circ}$; for pointing to the side, $M=22.93^{\circ}, S E M=0.78^{\circ}$; for pointing to the back, $M=26.69^{\circ}, S E M=1.32^{\circ}$. A post hoc contrast comparing accuracy in pointing to the front with that to all the other directions was significant $[F(1,47)=$ 35.54].

Mean response latency was $18.33 \mathrm{sec}$. There was no evidence for speed-accuracy tradeoff. (The correlation coefficient between absolute angular error and response latency was .48.)

These results suggest that both visually and proprioceptively learned orientations were preferred, indicating that both types of learned orientations were represented in spatial memory. The benefit for each type of learned orientation was equivalent, suggesting that both visual and proprioceptive representations were equally accessible.

These results exhibit a contrast to those from Shelton and McNamara (2001a). Participants in Shelton and McNamara's (2001a) experiments learned either a single orientation or multiple orientations of a room-sized spatial layout, and all orientations were learned visually. Oth-

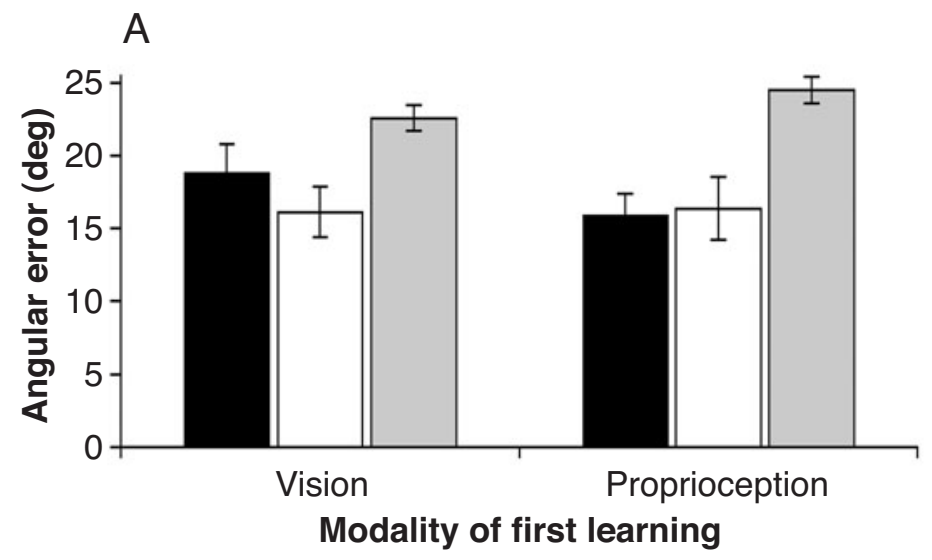

$\begin{array}{lll}\text { Visually learned } & \square \text { Proprioceptively } & \square \text { Novel } \\ \text { orientation } & \text { learned orientation } & \text { orientations }\end{array}$

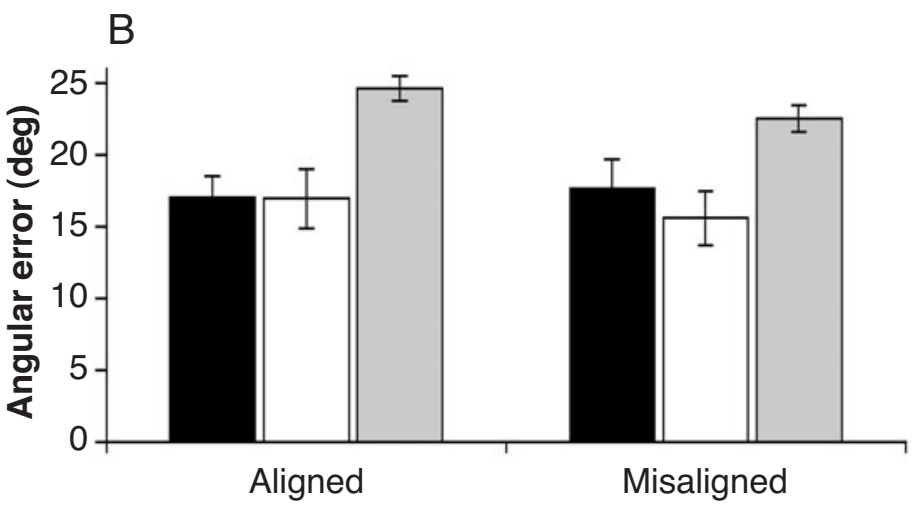

Alignment of visually learned orientation with walls

Figure 5. Mean absolute angular error in judgments of relative direction in Experiment 2 as a function of (A) type of orientation and order of learning and (B) type of orientation and alignment of the visually learned orientation with walls of the room. Error bars represent \pm 1 SEM. 
erwise, their experiments and the present experiment employed very similar materials and the identical procedure. A basic result from Shelton and McNamara's (2001a) experiments was that only the first learned orientation was accessible in spatial memory when multiple orientations were studied without any environmental cues. On the other hand, in the present study, both first and second learned orientations were equally preferred, independently of order of learning. This is depicted in Figure 5A, showing mean absolute angular error in pointing as a function of type of orientation and order of learning. Also, neither the main effect of order of learning nor the interactions including this variable were significant in the ANOVA. This contrast highlights the multimodal aspect of the spatial representations gained in the present experiment, suggesting the reason why both first and second learned orientations were preferred. Both were "first" learned orientations, because they were learned by different modalities.

With regard to the comparison between the present results and those of Shelton and McNamara (2001a), alignment of the visually learned orientation with the walls of the room is also an important factor. Shelton and McNamara (2001a) showed that there was no preference for a visually learned orientation that was not aligned with environmental structures (such as walls of a room), when those structures were available for spatial learning and another learned orientation (or other learned orientations) was aligned with them. In other words, Shelton and McNamara (2001a) showed that a misaligned orientation was preferred only when it was the sole learned orientation of an environment. On the basis of this finding, it could be speculated that the visually learned orientation in the present experiment would have not been represented in spatial memory when it was misaligned with the walls of the room. However, the results from this experiment revealed that the visually learned orientation was preferred even when it was misaligned with the walls. This is shown by mean absolute angular error in pointing, plotted in Figure 5B as a function of type of orientation and alignment. Accuracy in JRDs for the visually learned orientation was higher than that for novel orientations, regardless of alignment. Also, neither the main effect of alignment nor the interactions including this variable were significant in the ANOVA. This result suggests that different modalities yielded spatial representations separately: The visually learned orientation was preferred even when it was misaligned with the walls, because it was the one and only orientation learned visually.

In summary, Experiment 2 showed that both visually and proprioceptively learned orientations were represented in spatial memory. Both types of learned orientations were preferred during memory access independently of order of learning and alignment of the visually learned orientation with the walls of the room. These results suggest that multimodal representations of the environment were acquired by learning its different orientations visually and proprioceptively.

\section{GENERAL DISCUSSION}

The objective of the present study was to explore how spatial information from vision and proprioception is represented in spatial memory. The results suggest that (1) spatial memories for a room-sized layout acquired through each modality were orientation dependent and (2) multiple orientations of a layout learned by different modalities were accessible in spatial memory. The first finding is essentially the same as that from Presson et al.'s (1987) walk-no turn condition, providing strong support for their original result. The second finding was obtained independently of both order of learning and alignment of the visually learned orientation with the walls of the room, thereby making a marked contrast with the results from Shelton and McNamara's (2001a) experiments, in which participants visually learned multiple orientations of a layout.

In the experiments reported in the present article, participants' performance in JRDs always depended on the orientations experienced during spatial learning, regardless of the number of learned orientations and the modality of learning. In light of McNamara and colleagues' theoretical framework, these results can be interpreted to mean that participants selected two intrinsic reference axes, one based on visual learning and the other based on proprioceptive learning, and the layout was interpreted in terms of two different systems of spatial reference defined by each intrinsic axis. Each reference system alone was sufficient for spatial learning, as shown by the orientation-dependent performance in JRDs in Experiments 1 and 1A. Participants used both reference systems when both were available (Experiment 2), as evidenced by the preference for both types of learned orientations in JRDs. In addition, equivalent performance was observed in JRDs for each type of learned orientation throughout all the experiments, suggesting that both reference systems worked independently with neither enhancing nor interfering with the other.

It is noteworthy that the present findings coincide with those from previous work in which Shelton and McNamara's paradigm was employed (Shelton \& McNamara, 2001b, 2004). Shelton and McNamara (2001b) asked participants to manually reconstruct a table-top-sized display of seven objects from nonegocentric perspectives. They visually perceived the original display from their egocentric perspective and had no visual access to their reconstructed display. Results from JRDs indicated that performance for both the manually reconstructed orientation and the visually perceived orientation was better than that for novel orientations. ${ }^{5}$ Shelton and McNamara (2004) found the same pattern of results when participants verbally described a table-top-sized display of seven objects from nonegocentric perspectives. (Both the described orientation and the visually learned orientation were better than novel orientations in JRDs.) These results can also be interpreted to mean that participants selected two intrinsic reference axes, one based on visual 
learning and the other based on nonvisual (either tactile or verbal) learning, and the layouts were interpreted by both axes.

Our findings, together with the previous findings from Shelton and McNamara (2001b, 2004), are analogous to the recent findings from both neuropsychological studies on humans and neurophysiological studies on monkeys that have suggested the existence of multiple perceptual representations of space in parietal cortex (for a review, see Andersen, Snyder, Bradley, \& Xing, 1997; Colby \& Goldberg, 1999). Contrary to the traditional concept that information from different modalities is combined into an integrated representation of space (for a review, see Critchley, 1953; Hyvärinen, 1982), these recent studies have indicated that information from each modality forms an individual spatial representation. The results from both Experiment 2 in the present study and experiments in Shelton and McNamara (2001b, 2004) showed that participants represented the same environment twice in memory by using two intrinsic reference axes, each of which was linked to each modality of learning. These multiple memory representations were potentially separable, as suggested by the finding that the two intrinsic reference systems worked independently.

Alternatively, the results from Experiment 2 could be interpreted to mean that participants formed a single amodal representation of a layout in memory. This representation contained information about spatial relations among objects specified from two learned orientations, which were not distinguished in terms of the modalities through which they were learned. Although the present data do not unequivocally support the hypothesis of multiple memory representations of space, they are more favorable to this hypothesis than to the alternate hypothesis of a single amodal representation. The alternate hypothesis does not fit well with the results that visually and proprioceptively learned orientations were preferred independently of both order of learning and alignment of the visually learned orientation with the walls. If two learned orientations had not been distinguished with regard to the learning modalities, the results from Experiment 2 would have been more comparable to those from Shelton and McNamara's (2001a) experiments in which two orientations of a layout were learned visually. In those experiments, either the first learned orientation or an aligned orientation became preferred during memory access. As described above, however, participants in our Experiment 2 preferred both the second learned orientation and the visually learned orientation that was misaligned with the walls, which diverge from Shelton and McNamara's (2001a) results.

It should also be noted that each component of proprioception (proprioception proper, vestibular sense, and efference copy; see the definition of proprioception in the introduction) may have dissociable roles in spatial learning. For example, some previous studies have suggested that vestibular sense is more important for perception of angular displacements than that of linear dis- placements, whereas proprioception proper and efference copy are more important for perception of linear displacements than that of angular displacements (for a review, see Berthöz et al., 1999). It has also been suggested that proprioception proper and efference copy can predominate vestibular sense when all three of these are available for spatial learning (Mittelstaedt \& Mittelstaedt, 2001; see also Waller et al., 2004; Waller, Loomis, \& Steck, 2003). Because the present study did not attempt to look into individual contributions of each component to proprioceptive spatial learning, further efforts to separate them out will be required in the future.

In summary, the present study underscores the multimodal aspect of spatial memory, demonstrating that multiple orientations of an environment can be represented by spatial learning in different modalities. Our findings suggest that multiple representations of the same environment are formed in memory, and that each is represented by an intrinsic reference system based on each modality of learning. These multiple memory representations are potentially separate, and therefore there will be mechanisms of connecting them together through which multimodal understanding of space comes about. Further research on both the nature of spatial representations and the mechanisms of combining spatial information from different modalities in memory will extend our knowledge of the human spatial memory system.

\section{REFERENCES}

Andersen, R. A., Snyder, L. H., Bradley, D. C., \& Xing, J. (1997). Multimodal representation of space in the posterior parietal cortex and its use in planning movements. Annual Review of Neuroscience, 20, 303-330.

Berthöz, A., Amorim, M.-A., Glasauer, S., Grasso, R., Takei, Y., \& Viaud-Delmon, I. (1999). Dissociation between distance and direction during locomotor navigation. In R. G. Golledge (Ed.), Wayfinding behavior: Cognitive mapping and other spatial processes (pp. 328348). Baltimore: Johns Hopkins University Press.

Chance, S. S., Gaunet, F., Beall, A. C., \& Loomis, J. M. (1998). Locomotion mode affects the updating of objects encountered during travel: The contribution of vestibular and proprioceptive inputs to path integration. Presence: Teleoperators \& Virtual Environments, 7 , 168-178.

Colby, C. L., \& Goldberg, M. E. (1999). Space and attention in parietal cortex. Annual Review of Neuroscience, 22, 319-349.

Critchley, M. (1953). The parietal lobes. London: Arnold.

DiwadKar, V. A., \& McNamara, T. P. (1997). Viewpoint dependence in scene recognition. Psychological Science, 8, 302-307.

Dufour, A., Després, O., \& PebaYle, T. (2002). Visual and auditory facilitation in auditory spatial localization. Visual Cognition, 9, 741-753.

Franklin, N., Henkel, L. A., \& Zangas, T. (1995). Parsing surrounding space into regions. Memory \& Cognition, 23, 397-407.

HyväRINEN, J. (1982). The parietal cortex of monkey and man. Berlin: Springer-Verlag.

KLATZKY, R. L. (1999). Path completion after haptic exploration without vision: Implications for haptic spatial representations. Perception \& Psychophysics, 61, 220-235.

KLATZKY, R. L., \& LEDERMAN, S. J. (2003). Representing spatial location and layout from sparse kinesthetic contacts. Journal of Experimental Psychology: Human Perception \& Performance, 29, 310-325.

KLATZKY, R. L., LiPPA, Y., LOOMIS, J. M., \& GolledGe, R. G. (2002). Learning directions of objects specified by vision, spatial audition, or auditory spatial language. Learning \& Memory, 9, 364-367.

Klatzky, R. L., Loomis, J. M., Beall, A. C., Chance, S. S., \& 
Golledge, R. G. (1998). Spatial updating of self-position and orientation during real, imagined, and virtual locomotion. Psychological Science, 9, 293-298.

Loomis, J. M., Klatzky, R. L., Philbeck, J. W., \& Golledge, R. G. (1998). Assessing auditory distance perception using perceptually directed action. Perception \& Psychophysics, 60, 966-980.

Mardia, K. V., \& JUPP, P. E. (2000). Directional statistics. Chichester, U.K.: Wiley.

McNamara, T. P. (2003). How are the locations of objects in the environment represented in memory? In C. Freksa, W. Brauer, C. Habel, \& K. F. Wender (Eds.), Spatial cognition III: Routes and navigation, human memory and learning, spatial representation and spatial reasoning (pp. 174-191). Berlin: Springer-Verlag.

McNamara, T. P., Rump, B., \& Werner, S. (2003). Egocentric and geocentric frames of reference in memory of large-scale space. Psychonomic Bulletin \& Review, 10, 589-595.

Mittelstaedt, M.-L., \& MitteLstaedt, H. (2001). Idiothetic navigation in humans: Estimation of path length. Experimental Brain Research, 139, 318-332.

Mou, W., \& McNAMARA, T. P. (2002). Intrinsic frames of reference in spatial memory. Journal of Experimental Psychology: Learning, Memory, \& Cognition, 28, 162-170.

Presson, C. C., DeLANGe, N., \& HaZelrigg, M. D. (1987). Orientationspecificity in kinesthetic spatial learning: The role of multiple orientations. Memory \& Cognition, 15, 225-229.

Presson, C. C., Delange, N., \& Hazelrigg, M. D. (1989). Orientation specificity in spatial memory: What makes a path different from a map of a path? Journal of Experimental Psychology: Learning, Memory, \& Cognition, 15, 887-897.

PRESSON, C. C., \& HAZELRIGG, M. D. (1984). Building spatial representations through primary and secondary learning. Journal of Experimental Psychology: Learning, Memory, \& Cognition, 10, 723-732.

Rock, I. (1973). Orientation and form. New York: Academic Press.

Roskos-Ewoldsen, B., McNamara, T. P., Shelton, A. L., \& Carr, W. (1998). Mental representations of large and small spatial layouts are orientation dependent. Journal of Experimental Psychology: Learning, Memory, \& Cognition, 24, 215-226.

ShELTON, A. L., \& MCNAMARA, T. P. (1997). Multiple views of spatial memory. Psychonomic Bulletin \& Review, 4, 102-106.

Shelton, A. L., \& McNamara, T. P. (2001a). Systems of spatial reference in human memory. Cognitive Psychology, 43, 274-310.

Shelton, A. L., \& McNamara, T. P. (2001b). Visual memories from nonvisual experiences. Psychological Science, 12, 343-347.

Shelton, A. L., \& MCNamara, T. P. (2004). Spatial memory and perspective taking. Memory \& Cognition, 32, 416-426.

Sholl, M. J., \& Nolin, T. L. (1997). Orientation specificity in representations of place. Journal of Experimental Psychology: Learning, Memory, \& Cognition, 23, 1494-1507.

Waller, D., Loomis, J. M., \& Haun, D. B. M. (2004). Body-based senses enhance knowledge of directions in large-scale environments. Psychonomic Bulletin \& Review, 11, 157-163.

WALler, D., LoOMIS, J. M., \& STECK, S. D. (2003). Inertial cues do not enhance knowledge of environmental layout. Psychonomic Bulletin \& Review, 10,987-993.

Waller, D., Montello, D. R., Richardson, A. E., \& Hegarty, M. (2002). Orientation specificity and spatial updating of memories for layouts. Journal of Experimental Psychology: Learning, Memory, \& Cognition, 28, 1051-1063.
Werner, S., \& SCHMIDT, K. (1999). Environmental reference systems for large-scale spaces. Spatial Cognition \& Computation, 1, 447-473. Zacks, J. M., Mires, J., Tversky, B., \& Hazeltine, E. (2000). Mental spatial transformations of objects and perspective. Spatial Cognition \& Computation, 2, 315-332.

\section{NOTES}

1. It has been observed that sometimes the intrinsic axis corresponding to the second experienced orientation, not the one corresponding to the first experienced orientation, is selected to interpret the layout (Shelton \& McNamara, 2001a, Experiments 1 and 3). It has also been observed that two intrinsic axes corresponding to multiple experienced orientations (and all orientations were learned visually) are selected (Shelton \& McNamara, 1997, 2001a, Experiment 1). However, these cases largely result from having multiple learned orientations that are also aligned with salient environmental cues.

2. To assess the validity of the disorienting procedure, 7 participants were taken to the center of the room in the same way as described in Experiment 1 , and a caster chair was turned around several times by an experimenter. The participants were first asked to point to the door from which they entered the room while wearing a blindfold. After pointing, they were rotated once more and asked to take off the blindfold. They were then asked to point to the door again. Most of them pointed incorrectly to the door, except one participant in the first pointing and three participants in the second pointing. These participants reported that their correct pointing was based on pure guesswork, and they had no idea about the location of the door. In addition, circular standard deviations (Mardia \& Jupp, 2000) of both the first and second pointing were large $\left(77.12^{\circ}\right.$ and $60.72^{\circ}$, respectively), suggesting that there was no agreement among the participants about the direction of the door.

3. This procedure was included because participants' memories for the layouts were assessed by a scene recognition test as well. This test was conducted after JRDs in a similar way to previous studies (Diwadkar \& McNamara, 1997; Shelton \& McNamara, 2001b, 2004). Results from the scene recognition test were rather variable, and no clear effects were observed. Therefore, they are not reported in this article. A modified version of proprioceptive learning in which the participants had no visual information during walking was examined in Experiment $1 \mathrm{~A}$.

4. Another ANOVA was conducted in which type of orientation was replaced with imagined heading (from $0-315^{\circ}$ with $45^{\circ}$ intervals; $0^{\circ}$ corresponded to the visually learned orientation). The results from the two ANOVAs converged.

5. Performance for the manually reconstructed orientation was better than that for the visually perceived orientation. However, this difference was probably due to the design of the task, in which participants attended more to the manually reconstructed orientation than to the visually perceived orientation. A similar effect was observed in Mou and McNamara's (2002) experiments in which participants were instructed to attend to a nonegocentric orientation. (JRDs were more accurate for this attended orientation than for a visually perceived orientation.) The essential point here is that both the manually reconstructed orientation and the visually perceived orientation were more preferred than novel orientations.

(Manuscript received January 28, 2004; revision accepted for publication May 21, 2004.) 